Pure and Applied Mathematics Quarterly

Volume 6, Number 2

(Special Issue: In honor of

Michael Atiyah and Isadore Singer)

$439-456,2010$

\title{
Spectral Theory of Complete Riemannian Manifolds
}

\author{
Harold Donnelly*
}

\begin{abstract}
A survey is presented about the spectrum of the Laplace operator on noncompact Riemannian manifolds. Topics include manifolds with purely continuous spectrum, eigenvalues embedded in the continuum, and spectral stability.
\end{abstract}

Keywords: Ricci curvature, maximal volume growth, Cartan-Hadamard manifolds, Rellich lemma, Kato-Birman Theory.

\section{$0 \quad$ INTRODUCTION}

Let $M$ be a complete Riemannian manifold and $\Delta$ its Laplace operator acting on functions. In local coordinates $\Delta f=g^{-1 / 2} \frac{\partial}{\partial x_{i}}\left(g^{1 / 2} g^{i j} \frac{\partial f}{\partial x_{j}}\right)$. It follows that $\int_{M} f \Delta f=-\int_{M}|\nabla f|^{2}$, when $f$ is smooth and compactly supported. So $\Delta$ is formally self-adjoint. The completeness of $M$ guarantees that there is a unique self-adjoint extension to an unbounded operator $\Delta$ acting on $L^{2} M$. If $M$ is compact, then $L^{2} M$ admits an orthonormal basis consisting of eigenfunctions $\phi_{i}$ with eigenvalues $\lambda_{i}$, that is $\Delta \phi_{i}=-\lambda_{i} \phi_{i}$. There have been many works concerning the eigenvalues and their relationship to the geometry of the manifold. These studies pertain to upper and lower bounds for eigenvalues and asymptotics of eigenvalues. The spectral theory is less highly developed when $M$ is noncompact.

The present paper constitutes an exposition of some topics of interest in current research concerning spectral theory of the Laplacian for noncompact Riemannian

Received November 6, 2007.

*Research partially supported by NSF DMS 0504729 
manifolds. One source of inspiration is the mathematical physics surrounding the Schrödinger operator. Many of the problems and techniques arising in quantum mechanics have analogs in the setting of complete Riemannian manifolds. One particularly interesting aspect is the influence of curvature upon the nature of the spectrum.

Our first section concerns methods for showing that $M$ has no square integrable eigenfunctions under suitable hypotheses. The simplest example is Euclidean space $R^{n}$ with its flat metric. A technique discovered by Rellich [22], in his studies of domains in $R^{n}$, admits extensive development. It is applicable to asymptotically flat manifolds and manifolds with non-negative Ricci curvature and maximal volume growth. It eventually becomes clear that for more general Riemannian manifolds one should distinguish between isolated eigenvalues of finite multiplicity and eigenvalues embedded in the continuum.

In section two, there is a discussion of embedded eigenvalues with emphasis upon simply connected complete manifolds of negative curvature. A basic tool is a method initiated by Kato in his studies of the Schrödinger operator on $R^{n}$. The point is that the exponential map provides a diffeomorphism between a Cartan-Hadamard manifold $M^{n}$ and Euclidean space $R^{n}$. By working in geodesic polar coordinates and renormalizing the measure one finds a setting where the differential inequalities of Kato [19] are capable of generalization.

The final third section concerns the topic of spectral stability. This concept was first introduced by F. Xavier, [25]. One says that $M$ is spectrally stable if any sufficiently small compactly supported perturbation of the metric yields a unitarily equivalent Laplacian. The Kato-Birman theory, [2], of trace class perturbations may be applied to the heat kernel yielding stability of the absolutely continuous spectrum. To show absence of singular continuous spectrum the limiting absorption principle or Kato's theory of $H$-smoothing operators are relevant. Discrete eigenvalues are generally unstable and the Rellich methods, described in section one, are used to show their absence.

This article was solicited by the editors for the special issue dedicated to M. F Atiyah and I. M. Singer. It may therefore be appropriate to mention that much of the author's early work was concerned with the index theory of elliptic operators. In particular, he wrote a joint paper [1] with Atiyah and Singer. We proved a conjecture of Hirzebruch relating special values of Hecke $L$-functions 
to signature defects of Hilbert modular varieties. This certainly stimulated the author to pursue a more general study of spectral theory for noncompact Riemannian manifolds.

\section{Purely Continuous Spectrum}

Euclidean space with its standard flat metric is certainly the most elementary example of a complete Riemannian manifold. The spectral theorem is realized through application of the Fourier transform. Alternatively, one may exploit the rotational symmetry of the metric to represent the Laplacian as a direct sum of ordinary differential operators. It follows that the spectrum is purely continuous and consists of the half line $[0, \infty]$. There are no square integrable eigenfunctions.

A natural problem is to seek more general geometric reasons for the absence of point spectrum. One wants a method which includes $R^{n}$ as a basic case but is not reliant upon overly specialized hypotheses such as rotational symmetry. A fundamental tool in this regard is an integral identity first discovered by Rellich for domains in Euclidean space. Suppose that $D$ is a bounded $C^{1}$ domain in the complete Riemannian manifold $M^{n}$. Let $X_{i}$ denote the components of a vector field and $X_{i, j}$ the components of its covariant derivative. One has

Lemma 1.1. For any $u \in C^{2}(D) \cap C^{1}(\bar{D})$ we have

$\int_{D}\left(X_{i j}+X_{j, i}\right) u_{i} u_{j}-\int_{D} \operatorname{div} X|\nabla u|^{2}+2 \int_{D} X u \Delta u=2 \int_{\partial D} X u \frac{\partial u}{\partial \eta}-\int_{\partial D}|\nabla u|^{2} X \cdot \eta$

Here $\eta$ denotes the outward pointing normal vector.

Proof. A direct calculation gives the identity $2 X_{i, j} u_{i} u_{j}=\operatorname{div}\left(2 X u \nabla u-|\nabla u|^{2} X\right)-$ $2 X u \Delta u+\operatorname{div} X|\nabla u|^{2}$. Lemma 1.1 follows by integration over $D$.

To apply the Rellich lemma to eigenfunctions we assume that $\Delta u=-\lambda u$. Substitution and another partial integration yield

$$
\begin{aligned}
\int_{D}\left(X_{i j}\right. & \left.+X_{j, i}\right) u_{i} u_{j}+\int_{D}\left(\lambda u^{2}-|\nabla u|^{2}\right) \operatorname{div} X \\
& =2 \int_{\partial D} X u \frac{\partial u}{\partial \eta}+\int_{\partial D}\left(\lambda u^{2}-|\nabla u|^{2}\right) X \cdot \eta
\end{aligned}
$$


Exhaustion of $M$ by bounded domains and application of Federer's coarea formula yield the next result. It is a fundamental tool for the theorems appearing in [13] and the closely related paper by Escobar and Freire [15]:

Proposition 1.2. Let $u \in L^{2}\left(M^{n}\right)$ be a solution to $\Delta u=-\lambda u$. Suppose that $X$ is a $C^{1}$ vector field on $M^{n}$ with $|\nabla X| \leq c$, then

$$
\int_{M^{n}}\left(X_{i j}+X_{j, i}\right) u_{i} u_{j}=\int_{M^{n}}\left(|\nabla u|^{2}-\lambda u^{2}\right) \operatorname{div} X .
$$

Most of the applications follow by specializing to $X=\nabla f$, where $f$ is a $C^{2}$ function with bounded Hessian. The identity then becomes

$$
\int_{M} 2 f_{i, j} u_{i} u_{j}=\int_{M}\left(|\nabla u|^{2}-\lambda u^{2}\right) \Delta f
$$

If $M=R^{n}$, then one chooses $f(x)=\frac{1}{2} \sum_{i=1}^{n} x_{i}^{2}$ so that $\operatorname{Hess}(f)=I$ and $\Delta f=n$. So

$$
\int_{R^{n}} 2|\nabla u|^{2}=\int_{R^{n}}\left(|\nabla u|^{2}-\lambda u^{2}\right) n=0 .
$$

The last step follows by partial integration and the eigenfunction equation $\Delta u=$ $-\lambda u$. So $|\nabla u|=0$ and $u$ is constant. Since $u$ is square integrable $u \equiv 0$.

The previous proof that $R^{n}$ has purely continuous spectrum is capable of considerable generalization. This is in contrast with the more standard proof by Fourier transformation or separation of variables. The most immediate extension is

Proposition 1.3. Suppose that $M^{n}$ supports a convex function $f$ with bounded Hessian. If the Hessian is positive at a point and the Laplacian $\Delta f$ is constant, then $M^{n}$ admits no square integrable eigenfunctions.

The requirement that $\Delta f$ is constant is quite restrictive. Nevertheless, functions satisfying the hypotheses of Proposition 1.3 do exist on certain rotationally symmetric Riemannian manifolds.

The next step in extending the scope of the method is to assume only that $\Delta f$ is close to a constant. The main results of [13] and [15] use the following observation in combination with comparison theory. 
Proposition 1.4. Assume that $M^{n}$ supports a convex function $f$ with bounded Hessian. Suppose also that for some $b>0$ there exists $\epsilon=\epsilon\left(M^{n}, b\right)$ satisfying $f_{i, j} \geq(b-\epsilon) g_{i, j}$, and $|\Delta f-n| \leq \epsilon$, where $\epsilon$ is sufficiently small. Then $\Delta u=-\lambda u$ and $u \in L^{2} M$ only when $u \equiv 0$.

A typical application is to choose $f(r)=\frac{1}{2} r^{2}$ where $r$ is the geodesic distance from a point. To guarantee that $f$ is $C^{2}$ one assumes the exponential map, with the given basepoint, to be a global diffeomorphism. This gives

Theorem 1.5. Let $M^{n}$ be a complete Riemannian manifold with a pole. Suppose the radial curvature satisfies $|K| \leq \delta(1+r)^{-2}$, for $\delta$ sufficiently small. Then $M$ admits no point spectrum.

Proof. It follows from the Hessian comparison theorem [16] that $f(r)=\frac{1}{2} r^{2}$ satisfies Hess $f \geq(1-\epsilon) g$ and $|\Delta f-n| \leq \epsilon$, for sufficiently small $\delta$. The conclusion now follows from Proposition 1.4.

The paper [13] puts the previous theorem into a much more general framework. One constructs various rotationally symmetric manifolds, such as paraboloids, where Proposition 1.3 applies. These rotationally symmetric models are then perturbed to manifolds with a pole. The symmetry is perhaps removed but one can still apply comparison theory and Proposition 1.4.

The results outlined above require global hypotheses on the complete Riemannian manifold $M$. These hypotheses include the existence of a $C^{2}$ function $f$ which is convex at every point. To construct such an $f$ one typically assumes that the exponential map exp : $T_{p} M \rightarrow M$ is a diffeomorphism for some point $p \in M$. However, since $R^{n}$ is the basic model, one expects that absence of point spectrum should persist if the metric is perturbed arbitrarily on any compact set. This is certainly the case for $R^{n}$. One simply employs separation of variables outside the perturbation.

The papers [9] and [12] formulate hypotheses in a neighborhood of infinity which guarantee that the Laplacian has purely continuous spectrum. The hypotheses are given in terms of an exhaustion function $b(x)$. If $M^{n}=R^{n}$, note that $b(x)=\left(x_{1}^{2}+x_{2}^{2}+\cdots+x_{n}^{2}\right)^{1 / 2}$ satisfies (i) $|\nabla b|=1$, when $x \neq 0$, (ii) $\Delta b=(n-1) b^{-1}$, when $x \neq 0$, and (iii) Hess $b^{2}=2 g$, where $g$ denotes the flat metric. The idea is that if $M^{n}$ supports an exhaustion function where these 
properties hold in a weak or approximate sense, then $\Delta$ should have no point spectrum.

The first step is to derive certain weighted $L^{2}$ estimates. Suppose that $M$ admits a $C^{2}$ exhaustion function $b(x)$ that satisfies the following conditions in the complement of a compact set (i) $\left|\mathrm{Hess}^{2}-2 \mathrm{~g}\right|<\epsilon_{1}$, (ii) $c_{1} \leq|\nabla b| \leq c_{2}$, and (iii) $c_{1} r \leq b \leq c_{2} r$. Here $r=r(x)$ denotes the geodesic distance from a fixed basepoint $p \in M$. Under these conditions one has

Proposition 1.6. Assume that $u$ belongs to $C^{2} M \cap L^{2} M$ and moreover $\Delta u=$ $-\lambda u, \lambda>0$, in the complement of a compact set. Then for any positive integer $k, \int_{M} b^{k}\left(u^{2}+|\nabla u|^{2}\right)$ is finite.

The proof of Proposition 1.6 is given in [9]. One ingredient is the Rellich lemma which was described above. Note that $-\Delta$ is positive semidefinite. Harmonic square integrable functions exist only on manifolds of finite volume, because such functions are necessarily constant.

Carleman estimates were developed in [9]. By combining these with Proposition 1.6, one shows the absence of point spectrum. Suppose that in the complement of a compact set, one has for some $\epsilon>0$, (i) $\mid$ Hess $^{2}-2 \mathrm{~g} \mid \leq \mathrm{c}_{1} \mathrm{~b}^{-\epsilon}$, (ii) ||$\nabla b|-1| \leq c_{2} b^{-\epsilon}$, and (iii) $c_{3} r \leq b \leq c_{4} r$. Combining the Carleman estimates with the weighted $L^{2}$ estimates gives

Theorem 1.7. Suppose $u \in L^{2} M \cap C^{2} M$ satisfies $\Delta u=-\lambda u$ in $M-K, \lambda>$ 0 . We assume $K$ is a compact set and $M-K$ is connected. If $M$ admits an exhaustion function with the properties specified above, then $u \equiv 0$.

A manifold $M^{n}$, of dimension $n \geq 3$, with one end is asymptotically locally Euclidean if the sectional curvature decays faster than quadratically and geodesic balls have Euclidean volume growth. More precisely, one assumes (i) $|K| \leq$ $c_{1} r^{-2-\epsilon}$, for some $c_{1}>0$ and $\epsilon>0$, where $r$ is the geodesic distance for a basepoint $p$, and (ii) Geodesic balls have $\operatorname{Vol} B(p, t) \geq c_{2} t^{n}$, for some $c_{2}>0$. These spaces provide interesting examples where Theorem 1.7 applies.

The Mourre theory was used in [12] to develop the above work. Let $\epsilon(r)$ signify a function satisfying $\epsilon(r) \rightarrow 0$ as $r \rightarrow \infty$, where $r(x)$ denotes the geodesic distance from a basepoint $p \in M$. Assume that $M$ admits an exhaustion function with the following properties (i) $c_{1} r \leq b \leq c_{2} r$, for some positive constants $c_{1}$ and $c_{2}$, 
(ii) $1-\epsilon(r) \leq|\nabla b| \leq 1+\epsilon(r)$, (iii) $\mid$ Hess b $^{2}-2 \mathrm{~g} \mid \leq \epsilon\left(\mathrm{r}\right.$ ), and (iv) $\left|d \Delta b^{2}\right| \leq \epsilon(r)$. Here $d$ denotes the exterior derivative and $|T|$ is the pointwise norm of the tensor $T$. Combining the Mourre theory with weighted $L^{2}$ estimates gives

Theorem 1.8. Suppose $u \in L^{2} M \cap C^{2} M$ satisfies $\Delta u=-\lambda u$ on $M$, for some $\lambda>0$. If $M$ admits an exhaustion function with the properties just specified, then $u \equiv 0$.

Theorem 1.8 is applicable to certain manifolds of non-negative Ricci curvature. Suppose that the dimension of $M$ is at least three. Assume (i) Ricci $(M) \geq 0$, (ii) Vol $B(p, t) \geq c_{1} t^{n}$, and (iii) $|K| \leq c_{2} r^{-2}$, where $K$ denotes the sectional curvature. A consequence of Theorem 1.8 is that $\Delta$ has purely continuous spectrum. Construction of the required exhaustion functions requires sophisticated techniques. Their existence is due to Cheeger and Colding [4] and Colding and Minicozzi [6].

Note that Theorem 1.7 requires a specific decay rate $b^{-\epsilon}$, for some $\epsilon>0$, and for both $\mid$Hess $^{2}-2 \mathrm{~g} \mid$ and ||$\nabla b|-1|$. No such precise rate of decay is needed in Theorem 1.8, only that $\mid$Hess $^{2}-2 \mathrm{~g} \mid \rightarrow 0$ and ||$\nabla b|-1| \rightarrow 0$ as one approaches infinity, i.e. in the complement of compact sets. However, the hypotheses of Theorem 1.8 also include $\left|d \Delta b^{2}\right| \rightarrow 0$ at infinity. No estimate on the third derivatives is needed for Theorem 1.7. So Theorem 1.8 does not supersede Theorem 1.7.

\section{Absence of Embedded Eigenvalues}

Consider the hyperbolic space $H^{n}$ with its metric of constant curvature -1 . Since $H^{n}$ is simply connected, the exponential map provides a diffeomorphism between $H^{n}$ and $R^{n}$. Nevertheless, the negative curvature has interesting effects on the spectral theory of the Laplacian. The spectrum of $-\Delta$ on $L^{2} H^{n}$ consists of the half line $\left[\frac{1}{4}(n-1)^{2}, \infty\right)$ and is purely continuous. However, square integrable eigenfunctions occur even for compactly supported perturbations of the metric. One simply sews in a large Euclidean disc and applies the minimax principle. This elementary construction shows that arbitrarily many eigenvalues less than $\frac{1}{4}(n-1)^{2}$ may occur when the metric is perturbed on a compact set. Rather than showing purely continuous spectrum under decay conditions $K \rightarrow-1$, the sensible problem is to show absence of embedded eigenvalues $\lambda>\frac{1}{4}(n-1)^{2}$. 
Recall that in geodesic polar coordinates the metric of $H^{n}$ is given by $(d s)^{2}=$ $(d r)^{2}+g^{2}(r)(d \theta)^{2}$, with $g(r)=\sinh r$. The method of separation of variables applies to determine the spectral representation of the Laplacian $\Delta$. The spherical harmonics $Y_{k, j}(\theta)$, for $k \geq 0$ and $1 \leq j \leq q(k)$ form a complete orthonormal basis for $L^{2}\left(S^{n-1}\right)$. Each $Y_{k, j}(\theta)$ belongs to a $q(k)$ dimensional eigenspace of the spherical Laplacian with corresponding eigenvalue $\lambda_{k}$. One may expand

$$
\phi(r, \theta)=\sum_{k=0}^{\infty} \sum_{j=1}^{q(k)} \phi_{k, j}(r) Y_{k, j}(\theta)
$$

A computation using the local defining formula for $\Delta$ gives

$$
\Delta \phi=\sum_{k=0}^{\infty} \sum_{j=1}^{q(k)} \Delta_{k} \phi_{k, j}(r) Y_{k, j}(\theta)
$$

where $-\Delta_{k} \phi_{k, j}=-\phi_{k, j}^{\prime \prime}-(n-1) \frac{g^{\prime}}{g} \phi_{k, j}^{\prime}+\lambda_{k} g^{-2} \phi_{k, j}$. The prime denotes differentiation in $r$. Thus $\phi_{k, j} \in L^{2}\left((0, \infty), g^{n-1}(r) d r\right)$. So $\Delta$ is decomposed into a direct sum of the operators $\Delta_{k}$ with multiplicity $q(k)$.

We now renormalize the measure to Lebesque measure. Note that $\Delta_{k}$ is unitarily equivalent to $D_{k}=g^{(n-1) / 2} \Delta_{k} g^{(1-n) / 2}$ acting on $L^{2}((0, \infty), d r)$. A calculation yields $-D_{k} \psi=-\psi^{\prime \prime}+\left[\gamma(r)+\lambda_{k} g^{-2}\right] \psi$. Here $\gamma(r)=\left(\frac{n-1}{2}\right) f^{\prime \prime}+\left(\frac{n-1}{2}\right)^{2}\left(f^{\prime}\right)^{2}$ with $g=e^{f}$. It follows that the essential spectrum of $\Delta$ on $H^{n}$ consists of the half line $\left[\frac{1}{4}(n-1)^{2}, \infty\right)$.

One may apply comparison theory and Cheeger's inequality [3] to show that there are no eigenvalues below $\frac{1}{4}(n-1)^{2}$. Let $\mathcal{D}$ be a domain in $H^{n}$ and $r$ the geodesic distance from a basepoint $p \in H^{n}-\mathcal{D}$. Then

$$
\left|\int_{\mathcal{D}} \Delta r\right|=\left|\int_{\partial \mathcal{D}} \frac{\partial r}{\partial \eta}\right| \leq \operatorname{vol}(\partial \mathcal{D})
$$

Now $\Delta r=(n-1) \operatorname{cothr} \rightarrow(\mathrm{n}-1)$ as $p \rightarrow \infty$. So the ratio $\operatorname{vol}(\partial \mathcal{D}) / \operatorname{vol}(\mathcal{D}) \geq \mathrm{n}-1$. Cheeger's inequality now gives $\lambda_{1} \geq \frac{1}{4}(n-1)^{2}$.

Pinsky [21] studied more general rotationally symmetric metrics. If $n=2$, he gave decay conditions upon the Gauss curvature which guarantee the absence of embedded eigenvalues greater than $\frac{1}{4}(n-1)^{2}$. More specifically, Pinsky assumed that $K \rightarrow-1$ at infinity and $\int_{0}^{\infty}|K+1| d r<\infty$, along with $K \leq-1$ for sufficiently large $r$. He then proposed the problem of studying the same question without the hypothesis of a rotationally symmetric metric. 
Pinsky's problem was taken up by the author in [7]. The inspiration for the method came from the analogous work of Kato [19], when he studied the Schrödinger operator on $R^{n}$. Kato's idea is to regard $L^{2}\left(R^{n}\right)=L^{2}\left(R^{+}\right) \times$ $L^{2}\left(S^{n-1}\right)$ and to systematically exploit differential inequalities for $L^{2}\left(S^{n-1}\right)$ valued functions on $R$. This is still an ordinary differential equations method but the level of sophistication is more than for simple separation of variables.

We again work in geodesic polar coordinates. For simplicity details are given only for surfaces but the generalization to higher dimensions only requires some standard results about Jacobi fields. Let $M$ be a complete simply connected negatively curved surface. We endow $M$ with a global system of geodesic polar coordinates where the metric is given by $(d s)^{2}=(d r)^{2}+g^{2}(r, \theta)(d \theta)^{2}$. If $K$ denotes the Gauss curvature then $K$ satisfies $\partial^{2} g / \partial r^{2}+K g=0$.

The Laplacian is given by the next formula

$$
\Delta \psi=g^{-1} \frac{\partial}{\partial r}\left(g \frac{\partial \psi}{\partial r}\right)+g^{-1} \frac{\partial}{\partial \theta}\left(g^{-1} \frac{\partial \psi}{\partial \theta}\right)
$$

Define $w=g^{1 / 2} \psi$ and $H=g^{1 / 2}\left(-\Delta-\frac{1}{4}\right) g^{-1 / 2}$. Then one has

$$
H w=\frac{-\partial^{2} w}{\partial r^{2}}-g^{-2} \frac{\partial^{2} w}{\partial \theta^{2}}+\Lambda \frac{\partial w}{\partial \theta}+V w
$$

where

$$
\Lambda=2 g^{-3} \frac{\partial g}{\partial \theta}
$$

and

$$
V=-\frac{5}{4} g^{-4}\left(\frac{\partial g}{\partial \theta}\right)^{2}+\frac{1}{2} g^{-3} \frac{\partial^{2} g}{\partial \theta^{2}}+\frac{1}{2} g^{-1} \frac{\partial^{2} g}{\partial r^{2}}-\frac{1}{4} g^{-2}\left(\frac{\partial g}{\partial r}\right)^{2}-\frac{1}{4}
$$

Suppose that $K(r, \theta) \leq 0$ satisfies the following decay conditions, uniformly in $\theta$, (i) $\lim _{r \rightarrow \infty} r|K+1|=0$. (ii) $\int r|K+1| d r<d_{1}$, (iii) $\int e^{2 r}\left|K_{\theta}\right| d r<d_{2}$, (iv) $\int e^{2 r}\left|K_{\theta \theta}\right|<d_{3}$. It then follows from the method of asymptotic integrations that $\Lambda=0\left(e^{-2 r}\right)$ and $V=o\left(r^{-1}\right)$ as $r \rightarrow \infty$.

The method of Kato and its generalizations consist in exploiting suitable differential inequalities for cleverly chosen functionals. Suppose that $-\Delta \psi=$ $\left(E+\frac{1}{4}\right) \psi$ with $E>0$. Then $H w=E w$ and $\int\|w\|^{2} d r<\infty$. Let $w^{\prime}=\frac{\partial w}{\partial r}$ and define

$$
\mathcal{G}(r)=\left(w^{\prime}, w^{\prime}\right)-\left(g^{-2} w_{\theta}, w_{\theta}\right)+E(w, w)
$$


Here ( , ) is the global inner product on $L^{2}\left(S^{1}\right)$ and $\|\quad\|$ denotes the curresponding norm. If the above curvature decay conditions hold, then $\frac{d}{d r}(r \mathcal{G}(r)) \geq 0$ for $r>R_{0}$, and so $\mathcal{G}(r) \leq 0$ because it is integrable.

Define $w_{m}=r^{m} w$, for any $m \geq 0$. Then $w_{m}$ satisfies the ordinary differential equation

$$
w_{m}^{\prime \prime}-2 m r^{-1} w_{m}^{\prime}+g^{-2} w_{m, \theta \theta}+m(m+1) r^{-2} w_{m}-\Lambda w_{m, \theta}+(E-V) w_{m}=0
$$

Let

$\mathcal{L}(m, r)=\left(w_{m}^{\prime}, w_{m}^{\prime}\right)+\left(E-E R_{0} r^{-1}+m(m+1) r^{-2}\right)\left(w_{m}, w_{m}\right)-\left(g^{-2} w_{m, \theta}, w_{m, \theta}\right)$

Under the assumed curvature decay hypotheses $\frac{d}{d r}\left(r^{2} \mathcal{L}(m, r)\right)>0$ for $m>m_{0}$ and $r>R_{1}>R_{0}$. Examination of the formula defining $\mathcal{L}(m, r)$ shows that for some $R_{2}>R_{1}$ there exists an $m_{1}>m_{0}$ so that $\mathcal{L}\left(m_{1}, R_{2}\right)>0$. Since $r^{2} \mathcal{L}\left(m_{1}, r\right)$ is increasing, we must have $\mathcal{L}\left(m_{1}, r\right)>0$, for all $r \geq R_{2}$.

On the other hand, we may choose $R_{3}>R_{2}$ so that $-E R_{0} r^{-1}+m_{1}\left(2 m_{1}+\right.$ 1) $r^{-2}<0$ if $r \geq R_{3}$. Since $\int\|w\|^{2}$ is finite, a suitable $R_{3}$ will also satisfy $\left(w^{\prime}, w\right)\left(R_{3}\right)<0$. Note that

$R_{3}^{-2 m_{1}} \mathcal{L}\left(m_{1}, R_{3}\right)=\left\|w^{\prime}+\frac{m w}{R_{3}}\right\|^{2}+\left(E-E R_{0} R_{3}^{-1}+m(m+1) R_{3}^{-2}\right)\|w\|^{2}-\left(g^{-2} w_{\theta}, w_{\theta}\right)$

Consequently, $R_{3}^{-2 m_{1}} \mathcal{L}\left(m_{1}, R_{3}\right) \leq \mathcal{G}\left(R_{3}\right) \leq 0$. This contradicts the result of the previous paragraph that $\mathcal{L}\left(m_{1}, R_{3}\right)>0$.

In summary, we have proved

Theorem 2.1. Let $M$ be a complete simply connected negatively curved surface. Suppose that the Gauss curvature satisfies (i) $\lim _{r \rightarrow \infty} r|K+1|=0$, (ii) $\int r \mid K+$ $1 \mid d r<d_{1}$ (iii) $\int e^{2 r}\left|K_{\theta}\right| d r<d_{2}$, and (iv) $\int e^{2 r}\left|K_{\theta, \theta}\right| d r<d_{3}$. Then $-\Delta$ has no eigenvalue greater that $1 / 4$.

Theorem 2.1 generalizes in a straightforward way to manifolds of dimension $n \geq$ 2. One need only utilize the technique of Jacobi fields and suitable theorems for systems of ordinary differential equations. The decay conditions are formulated in terms of sectional curvature and its covariant derivatives. One concludes that $-\Delta$ has no eigenvalues $\lambda>\frac{1}{4}(n-1)^{2}$. Precise statements and proofs are given in $[7]$. 
Most differential geometers find the hypotheses involving covariant derivatives of curvature to be unappealing. It is preferable to assume only that $K \rightarrow-1$ at a prescribed rate. This improvement was achieved in the paper [10]. Note that the $\theta$ dependence is introduced when one renormalizes the measure to get $H=$ $g^{1 / 2}(-\Delta-1 / 4) g^{-1 / 2}$, because $g=g(r, \theta)$. The first step is to simply renormalize by $e^{r / 2}$, so that $\bar{H}=\bar{g}^{1 / 2}\left(-\Delta-\frac{1}{4}\right) \bar{g}^{-1 / 2}$, with $\bar{g}=e^{r}$. The operator $\bar{H}$ is less suitable for Kato's method and various complications ensue when choosing the correct generalizations of the functionals $\mathcal{G}(r)$ and $\mathcal{L}(m, r)$. After some additional effort one proves

Theorem 2.2. Let $M^{n}$ be a complete simply connected negatively curved manifold whose sectional curvatures converge to -1 at infinity. Assume the decay conditions (i) $\int_{0}^{\infty} t^{\beta}|K+1| d t<d_{1}$ and (ii) $\lim _{t \rightarrow \infty} t^{\beta}|K+1|=0$, uniformly in the spherical variable and with $\beta>2$. Then $-\vec{\Delta}^{\infty}$ has no eigenvalues greater than $(n-1)^{2} / 4$.

In a recent preprint [20], Kumura improved the last result by assuming only $t^{\beta}|K+1| \rightarrow 0$, with $\beta>1$. He achieves further generality by using the exponential map from an embedded submanifold rather than just the exponential map from a point. The submanifold is assumed to be the boundary of a compact domain and to have non-negative second fundamental form with respect to an outward pointing normal vector. Kumura also provides an example showing that his result is sharp. Namely, there exists a manifold with $|K+1|=0\left(r^{-1}\right)$ and with large convex geodesic spheres where $-\Delta$ has an eigenvalue $1+(n-1)^{2} / 4$. These examples have rotational symmetry, so that separation of variables is applicable. The resulting ordinary differential operators are of the type considered by Wigner and von Neumann in their celebrated construction of Schrödinger operators with eigenvalues embedded in the continuum.

If one does not require large convex geodesic spheres, then there are examples where $K \equiv-1$ outside a compact set and $-\Delta$ has arbitrarily large eigenvalues. Let $g(r)$ be a function satisfying $g(0)=0, g^{\prime}(0)=1$, and $g(r)=e^{-r}$, for $r \geq 1$. Let $M$ be a Riemannian manifold diffeomorphic to $R^{2}$ with metric $d s^{2}=d r^{2}+$ $g^{2}(r) d \theta^{2}$. Separation of variables shows that $\Delta$ is unitarily equivalent to a direct sum of ordinary differential operators

$$
D_{n} \psi=\psi^{\prime \prime}+\left[w(r)-n^{2} g^{-2}(r)\right] \psi
$$


where $w(r)=-\frac{1}{2} h^{\prime \prime}(r)-\frac{1}{4}\left(h^{\prime}(r)\right)^{2}$ and $g(r)=\exp (h(r))$. Thus, if $r \geq 1$, one has $-D_{n} \psi=-\psi^{\prime \prime}+\left[\frac{1}{4}+n^{2} e^{2 r}\right] \psi$. A theorem of Titchmarsh and Weyl [22] states that operators of the form $\frac{-d^{2}}{d r^{2}}+q(r)$, with $q(r) \rightarrow \infty$ as $r \rightarrow \infty$, have empty essential spectrum. Thus, when $n \neq 0,-D_{n}$ has pure point spectrum consisting of eigenvalues $\lambda_{m}(n)$, where $\lambda_{m}(n) \rightarrow \infty$ as $m \rightarrow \infty$. On the other hand $-D_{0}$ has no eigenvalues greater than $1 / 4$ because $-D_{0}=-d^{2} / d r^{2}+1 / 4$, outside the compact set $r \leq 1$. So in these examples $-\Delta$ has continuous spectrum consisting of the interval $[1 / 4, \infty)$. Moreover, $-\Delta$ has arbitrarily large eigenvalues embedded in the continuum.

Methods extending the ideas of Kato are also applicable to asymptotically flat manifolds. The first such results were found by Leon Karp [18] who gave decay conditions on $K$ and $K_{\theta}$ which guarantee no eigenvalues for asymptotically flat surfaces. Karp notes that the methods of Kato are in fact closely related to the Rellich identities described in the first section of this paper. The author [8] showed that more stringent decay conditions on $K$ alone guarantee purely continuous spectrum. These works both assume that $M$ is a simply connected complete surface with $K \leq 0$ globally. The more recent work of Kumura [20] reaches the same conclusion in all dimensions with weaker decay assumptions on $K$ than those of [8].

\section{Spectral Stability}

The concept of spectral stability was introduced by F. Xavier in an unpublished paper [25]. It was subsequently developed in joint work with the present author [14]. Recall that two self-adjoint operators $A_{1}, A_{2}$ acting on Hilbert spaces $H_{1}, H_{2}$, respectively, are said to be unitarily equivalent if there exists a unitary isomorphism $U: H_{1} \rightarrow H_{2}$ satisfying $U A_{1}=A_{2} U$, where $U$ preserves the domains of $A_{1}$ and $A_{2}$. The fundamental concept is

Definition 3.1. Let $(M, g)$ be a complete Riemannian manifold. We say that $g$ is spectrally stable if for any compactly supported two tensor $h$, there is a $\delta(h)>0$, so that $\Delta_{g}$ is unitarily equivalent to $\Delta_{g+\gamma h}$, for any $|\gamma|<\delta(h)$.

One notes that $g+\gamma h$ is positive definite for sufficiently small $\delta(h)$.

Let $A$ be a self adjoint operator acting on a Hilbert space $H$. In general, $A$ may be unbounded. The spectral theorem guarantees that $A$ is unitary equivalent to a 
multiplication operator. More precisely there is a collection $\mu_{\alpha}$ of Borel measures supported on the real line $R$ so that $A$ is unitarily equivalent to multiplication by $x$ on the Hilbert space $\sum_{\alpha} L^{2}\left(\mu_{\alpha}\right)$. Here $x$ is the standard coordinate on the real line $R$. According to the Lebesque decomposition theorem we may decompose each Borel measure as $\mu_{\alpha}=\mu_{\alpha, 1}+\mu_{\alpha, 2}+\mu_{\alpha, 3}$. Here $\mu_{\alpha, 1}$ is the absolutely continuous part of $\mu_{\alpha}, \mu_{\alpha, 2}$ is the singular continuous part of $\mu_{\alpha}$, and $\mu_{\alpha, 3}$ is the discrete part of $\mu_{\alpha}$. The absolutely continuous, singular continuous, and discrete part of $A$ correspond to the decomposition $\sum_{\alpha} L^{2}\left(\mu_{\alpha}\right)=\sum_{\alpha} L^{2}\left(\mu_{\alpha, 1}\right)+\sum_{\alpha} L^{2}\left(\mu_{\alpha, 2}\right)+$ $\sum_{\alpha} L^{2}\left(\mu_{\alpha, 3}\right)$.

Our approach to spectral stability of Riemannian manifolds entails separate consideration of the absolutely continuous, singular continuous, and discrete spectrum. The absolutely continuous spectrum is the most tractable. Application of the work of Birman and Kato [2] shows its stability for any complete Riemannian manifold. The singular continuous spectrum is more difficult. One may employ either the limiting absorption principle or $H$-smoothing operators [22]. These methods seem to be effective in special cases only. For the point spectrum one distinguishes between isolated eigenvalues and eigenvalues embedded in the continuum. The minimax principle is often applicable to give a lower bound on the spectrum and thus preclude the existence of small eigenvalues below the essential spectrum. Embedded eigenvalues are handled by the methods of Kato and Rellich as outlined in the earlier sections of this paper.

We now explain the stability of the absolutely continuous spectrum. Let $A$ and $B$ denote positive self adjoint operators acting on a Hilbert space $H$. Suppose that for some $t>0$, the difference $\exp (-t A)-\exp (-t B)$ is a trace class operator. Then the absolutely continuous part of $A$ is unitarily equivalent to the absolutely continuous part of B by the work of Birman and Kato [2]. One applies this result to the Laplacians $\Delta_{1}, \Delta_{2}$ of two metrics that agree outside a compact set and are both complete. The corresponding Hilbert spaces are identified by conjugation. Duhamel's principle and the finite propagation speed for the wave equation show that the difference of the heat kernels $\exp \left(t \Delta_{1}\right)-\exp \left(t \Delta_{2}\right)$ is trace class. Recall that for $R^{n}$ the heat kernel is given by $(4 \pi t)^{-n / 2} \exp \left(-|x-y|^{2} / 4 t\right)$. Rapid decay off the diagonal is a very general phenomenon for complete Riemannian manifolds. Cheeger, Gromov, and Taylor [5] wrote the heat kernel as a Fourier transform using the wave kernel. This gave rapid decay in the $L^{2}$ sense of the heat kernel 
for any complete Riemannian manifold. Alternatively, one may employ powers of the resolvent kernel [25] instead of the heat kernel.

There is at present no such general technique for dealing with the discrete spectrum or the singular continuous spectrum. The main positive result is

Theorem 3.2. Any Riemannian symmetric space of noncompact type is spectrally stable.

We proceed to outline two proofs of Theorem 3.2. The first works in full generality and is developed from the author's paper [11]. It relies upon the function theory of symmetric spaces as expounded by Helgason [17] and Harish-Chandra. This consists of the sophisticated use of representation theory for semisimple groups. There is little prospect of generalization to complete Riemannian manifolds even if one assumes simple connectedness and variable negative curvature. A second method initiated by Xavier [25] has promise for greater scope, although it does not yet cover all cases of Theorem 3.2. It is tied to the Rellich identities of the first section of this paper. Convexity of functions is applicable not just to showing absence of point spectrum but also to ruling out the presence of singular continuous spectrum. However, for the singular continuous spectrum, certain fourth order conditions intervence which pose great difficulties.

Let $\left(M, g_{0}\right)$ be a Riemannian symmetric space of noncompact type. The Fourier analysis developed by Helgason shows that $-\Delta_{g_{0}}$ is unitarily equivalent to multiplication by $\langle\rho, \rho\rangle+x^{2}$ on $L^{2}\left(R^{+}, d x, \mathcal{M}\right)$, where $\mathcal{M}$ denotes a Hilbert space of countably infinite dimension. Here $\rho$ denotes half the sum of the positive restricted roots. This includes the case of Euclidean space $R^{n}$ with $\rho=0$. In general there is an important qualitative change due to the gap in the spectrum of the interval $[0,\langle\rho, \rho\rangle]$. For hyperbolic space $H^{n}$, one has $\langle\rho, \rho\rangle=(n-1)^{2} / 4$, which explains the connection to the previous section of this paper. The spectral resolution for hyperbolic space may be obtained by separation of variables and reduction to ordinary differential equations. More sophisticated methods are needed to treat symmetric spaces of higher rank.

A major ingredient in the first proof of Theorem 3.2 is the following result most of which was already established in [11]:

Theorem 3.3. Let $(M, g)$ be obtained by a compactly supported perturbation of the metric on a Riemannian symmetric space $\left(M, g_{0}\right)$. Then the continuous part 
of $\Delta_{g}$ is unitarily equivalent to $\Delta_{g_{0}}$. Moreover, $\Delta_{g}$ has at most finitely many eigenvalues which are all less than $\langle\rho, \rho\rangle$.

Proof. (i) As noted above, $\exp \left(t \Delta_{g}\right)$ is a trace class perturbation of $\exp \left(t \Delta_{g_{0}}\right)$. So by the Birman-Kato theory the absolutely continuous part of $\Delta_{g}$ is unitarily equivalent to $\Delta_{g_{0}}$, which is purely absolutely continuous.

(ii) The operator $\Delta_{g}$ has no singular continuous spectrum. This follows by analytically continuing the resolvent $\left(\Delta_{g}+z\right)^{-1}$ as a perturbation of $\left(\Delta_{g_{0}}+z\right)^{-1}$. One establishes the existence of boundary values on $R^{+}$when the resolvents are restricted to weighted $L^{2}$ spaces. The limiting absorption principle is then applicable.

(iii) There are no embedded eigenvalues $\lambda \geq\langle\rho, \rho\rangle$ and finitely many eigenvalues less that $\langle\rho, \rho\rangle$. Fourier analysis and unique continuation show the absence of embedded eigenvalues. One extends the Fourier transform of a hypothetical eigenfunction with $\lambda>\langle\rho, \rho\rangle$ to the complex plane and employs the Paley-Wiener theorem. Dirichlet Neumann bracketing shows that there are finitely many eigenvalues less than $\langle\rho, \rho\rangle$. A similar comparison with an exterior problem also shows the absence of eigenvalues $\lambda \geq\langle\rho, \rho\rangle$.

The proof of Theorem 3.2 is now reduced to obtaining the required lower bound of the spectrum for sufficiently small perturbations of the metric. Of course, by sewing in a large Euclidean disc and applying the minimax principle, it is easily seen that suitable large but compactly supported perturbations of the metric give finitely many eigenvalues less than $\langle\rho, \rho\rangle$. Consider the Cartan decomposition $G=K A K$. The induced volume element on the Weyl chamber is $w=\prod_{\alpha \in \sum^{+}}(\sinh \alpha(H))^{m_{\alpha}}$, where $\sum^{+}$denotes the set of restricted positive roots. If $\rho=\frac{1}{2} \sum_{\alpha \in \sum^{+}} m_{\alpha} \alpha$ denotes half the sum of the positive restricted roots, let $\vec{n}=\vec{\nabla} \rho /|\vec{\nabla} \rho|$. If $\phi$ is of compact support, then integration by parts in the Weyl chamber gives

$$
|\rho|^{2}\left[\int \phi^{2} w+\int w \phi^{2}\left(\frac{\vec{n} w}{2|\rho| w}-1\right)\right] \leq \int|\nabla \phi|^{2} w
$$

The integration runs over the Weyl chamber which contains the support of $\phi$. Standard properties of $\sum^{+}$guarantee that $\vec{n} w / 2|\rho| w \geq 1$. This provides the stability. Of course, the difference $\vec{n} w / 2|\rho| w-1$ decays to zero at infinity, but 
one is only considering compactly supported perturbation of the metric. For the special case of hyperbolic space, the above calculation takes place in geodesic polar coordinates. One has $w=(\sinh r)^{n-1}$ and the stabilizing term is just $\operatorname{coth} r-1$.

The second approach to the proof of Theorem 3.2 relies upon the work of Xavier in [24] and [25]. He extends the ideas of Rellich, outlined in the first section of this paper, and employs Kato's theory of $H$-smoothing operators to prove the following result:

Theorem 3.4. Let $M$ be a complete Riemannian manifold with metric $g_{0}$. Suppose that $M$ supports a strictly convex function $f$ which has bounded Laplacian and gradient and satisfies $\Delta^{2} f \leq 0$. Assume that for all $\phi \in C_{0}^{\infty}(M)$,

$$
\int_{M} \operatorname{Hessf}(\nabla \phi, \nabla \phi) \geq \int_{\mathrm{M}} \eta \phi^{2}
$$

where $\eta$ is a positive continuous function. Then $\Delta_{g_{0}}$ has purely absolutely continuous spectrum. Moreover, if $g$ is a sufficiently small compactly supported perturbation of $g_{0}$, then $\Delta_{g}$ has purely absolutely continuous spectrum.

Since the absolutely continuous spectrum is known to be stable, Theorem 3.4 is potentially a valuable tool for proving spectral stability in greater generality, where the methods of Theorem 3.3 are not available. The difficulty comes in satisfying the hypothesis $\Delta^{2} f \leq 0$. For symmetric spaces of noncompact type, one takes $f$ to be an infinite sum of Busemann functions plus a coordinate function on $A$. Here $G=K A N$ is the Iwasawa decomposition. Hardy's inequality is applied on an abelian $B \subseteq N$ which requires the condition $\operatorname{dim} B \geq 3$. So the method fails for the hyperbolic spaces $H^{2}$ and $H^{3}$. There are other exceptional cases. For the $f$ just described $\Delta f$ is constant and thus $\Delta^{2} f=0$. Although one would like to extend the technique to certain manifolds of variable negative curvature it seems hard to find suitable $f$ with $\Delta^{2} f \leq 0$.

We close with some remarks concerning spectral instability. It follows from Weyl's asymptotic formula that any compact Riemannian manifold is spectrally unstable. Namely $\operatorname{vol}(M)=c_{n} \lim _{\lambda \rightarrow \infty} \lambda^{-n / 2} N(\lambda)$ where $N(\lambda)$ denotes the number of eigenvalues less than $\lambda$, and $\operatorname{vol}(M)$ denotes the volume of $M$. Small perturbations of the metric can certainly change the volume. More generally, elementary perturbation theory indicates that isolated eigenvalues of finite multiplicity are 
shifted by small compactly supported perturbations in the metric. Xavier [25] showed that cylindrical manifolds $M \times R$ are spectrally unstable. Here $M$ is compact and $R$ denotes the real line. The cylindrical manifolds have purely absolutely continuous spectrum with finite but nonconstant multiplicity. The thresholds are determined by the eigenvalues of the compact factor. Separation of variables shows that small compactly supported changes in the metric can create infinitely many embedded eigenvalues, with at most finitely many corresponding to each threshold.

\section{REFERENCES}

[1] Atiyah, M.F., Donnelly, H., and Singer, I.M., Eta invariants, signature defects of cusps, and values of L-functions, Annals of Math., 118 (1983), 131-177.

[2] Birman, M.S., Existence conditions for wave operators, Izv. Akad. Nauk SSSR, Ser. Mat., 27 (1973), 883-906.

[3] Cheeger, J., A lower bound for the smallest eigenvalue of the Laplacian, Bochner volume, Princeton University Press, 1970, 195-199.

[4] Cheeger, J. and Colding, T., Lower bounds on Ricci curvature and the almost rigidity of warped products, Annals of Math., 144 (1996), 189-237.

[5] Cheeger, J., Gromov, M., and Taylor, M., Finite propagation speed, kernel estimates for functions of the Laplace operator, and the geometry of complete Riemannian manifolds, J. Differential Geometry, 17 (1982), 15-53.

[6] Colding, T. and Minicozzi, W., Large scale behavior of kernels of Schrödinger operators, Amer. J. Math., 119 (1997), 1355-1398.

[7] Donnelly, H., Eigenvalues embedded in the continuum for negatively curved manifolds, Michigan Math. J., 28 (1981), 53-62.

[8] Donnelly, H., Embedded eigenvalues for asymptotically flat surfaces, Proceedings of Symposia in Pure Mathematics, 54 (1993), 169-177.

[9] Donnelly, H., Exhaustion functions and the spectrum of complete Riemannian manifolds, Indiana Univ. Math. J., 46 (1997), 505-527.

[10] Donnelly, H., Negative curvature and embedded eigenvalues, Math. Z., 203 (1990), 301-308.

[11] Donnelly, H., Spectral geometry for certain noncompact Riemannian manifolds, Math. Z., 169 (1979), 63-76.

[12] Donnelly, H., Spectrum of the Laplacian on asymptotically Euclidean spaces, Michigan Math. J., 46 (1999), 101-111.

[13] Donnelly, H. and Garofalo, N., Riemannian manifolds whose Laplacians have purely continuous spectrum, Math. Ann., 293 (1992), 143-161.

[14] Donnelly, H. and Xavier, F., Spectral stability of symmetric spaces, Math. Z., 253 (2006), 655-658.

[15] Escobar, J. and Freire, A., The spectrum of the Laplacian of manifolds of positive curvature, Duke Math. J., 65 (1992), 1-21. 
[16] Greene, R. and Wu, H., Function theory on manifolds which possess a pole, Springer Lecture Notes in Math., Vol 699, Berlin, Heidelberg, N.Y., 1979.

[17] Helgason, S., Functions on symmetric spaces, Proceedings of symposia in pure mathematics, 26 (1973), American Mathematical Society, Providence, 1973, 101-146.

[18] Karp, L., Noncompact Riemannian manifolds with purely continuous spectrum, Michigan Math. J., 31 (1984), 339-347.

[19] Kato, T., Growth properties of solutions of the reduced wave equation with a variable coefficient, Comm. Pure and Applied Math., 12 (1959), 403-426.

[20] Kumura, H., Geometry of an end and the absence of eigenvalues in the essential spectrum, arXiv:math. DG/0505557, v.3, 3 Jul 2005.

[21] Pinsky, M., The spectrum of the Laplacian on a manifold of negative curvature II, J. Differential Geometry, 14 (1979), 609-620.

[22] Reed, M. and Simon, B., Methods of Modern Mathematical Physics, Volumes I-IV, Academic Press, N.Y., 1972.

[23] Rellich, F., Über das asymptotische Verhalten der Lösungen von $\Delta u+\lambda u=0$ in unendlichen Gebieten, Jahresber. Dtsch. Math. -Ver., 53 (1943), 57-65.

[24] Xavier, F., Convexity and absolute continuity of the Laplace Beltrami operator, Math. Annalen, 282 (1988), 579-585.

[25] Xavier, F., Persistence of absolute continuity and spectral stability of hyperbolic spaces, unpublished paper.

Harold Donnelly

Department of Mathematics

Purdue University

West Lafayette, IN 47907

E-mail: hgd@math.purdue.edu 